\title{
Influence of Imaging Parameters and Specimen Thinning on Strain Measurements in Au/Ni MBE Multilayers by HREM Image Processing
}

\author{
Pascale Bayle-Guillemaud and Jany Thibault $\left({ }^{*}\right)$ \\ CEA-Grenoble/Département de Recherche Fondamentale sur la Matière Condensée/SP2M, \\ 17 rue des Martyrs, 38054 Grenoble Cedex 9, France
}

(Received November 25, 1996; accepted March 25, 1997)

PACS.61.16.Bg - Transmission, reflection and scanning electron microscopy (including EBIC)

PACS.68.35.-p - Solid surfaces and solid-solid interfaces

PACS.07.05.Pj - Image processing

\begin{abstract}
Experimental distortion profiles have been measured in strained $\mathrm{Au} / \mathrm{Ni} \mathrm{MBE}$ multilayers using a quantitative analysis of the HREM micrographs. In order to study the reliability of these measurements, similar analysis have been made on simulated HREM images of $\mathrm{Au} / \mathrm{Ni}$ interfaces. It is found that the measured profiles are reliable only on a limited range of experimental conditions i.e. microscope defocus and specimen thicknesses. Furthermore, it will be shown that the presence of a mixed layer introduced by the ion beam thinning on both sides of the cross section specimen affects considerably the shape of the strain profile recorded on thin areas.
\end{abstract}

\section{Introduction}

$\mathrm{Au} / \mathrm{Ni}$ strained multilayers have been investigated by HREM. The lattice parameters of $\mathrm{Au}$ and $\mathrm{Ni}$ are $0.40789 \mathrm{~nm}$ and $0.35252 \mathrm{~nm}$ respectively. This gives a lattice misfit of $m=\left(a_{\mathrm{Au}}-a_{\mathrm{Ni}}\right) / a_{\mathrm{Ni}}=15.6 \%$. In a pseudomorphic regime (i.e. coherent interfaces), the $\mathrm{Ni}$ lattice is strained in tension on the (002)Au growth plane and consequently a tetragonal distortion (in compression) occurs perpendicularly to the interface. HREM observations have shown that the growth remains coherent until a Ni thickness layer of 4 monolayers (ML). Distortion profiles along the growth direction were measured using image processing on HREM micrographs. In Section 2, we summarize the method used to quantify this distortion and the experimental results already presented in details in reference [1]. The distortion profiles were measured for Ni layers thinner than $5 \mathrm{ML}$ (i.e. in the coherent growth regime).

The high resolution electron microscopy (HREM) images being generally non linear with respect to the atomic column position, they must be validated on simulated HREM images of $\mathrm{Au} / \mathrm{Ni}$ interfaces and by a subsequent comparison between the strain profiles deduced from the experimental and simulated images. In Section 3 we then discuss the influence of the sample thickness and microscope defocus on the contrast of the HREM images of the Au/Ni interfaces. In Section 4, we discuss the effect of the mixing between the two $\mathrm{Au}$ and $\mathrm{Ni}$ components

$\left.{ }^{*}\right)$ member of CNRS 
which might be introduced at the two specimen surfaces either during the ion-milling used in sample preparation or by the incident electron beam. This affects also the position of the dots with respect to the atomic column position.

Furthermore, it is known that the measurement of experimental distortion profile might be largely affected by relaxation at free surfaces due to the thinning of the specimen: the stress field being no longer planar [2]. This factor might be important when the multilayer is made with layers of the same thickness. It will be shown in Section 5 that in our $\mathrm{Au} / \mathrm{Ni}$ multilayers with thin Ni layers, this factor is not predominant.

\section{Au/Ni MBE Multilayers: Description and Observation}

\subsection{Description}

The $\mathrm{Au} / \mathrm{Ni}$ multilayers were grown at room temperature by molecular beam epitaxy on a [001] $\mathrm{MgO}$ substrate [3]. A $1 \mathrm{~nm}$ thick iron layer stabilizes a [001] growth direction. Multilayers with a number of $(002)_{\mathrm{Ni}}$ planes varying from 1 to 4 separated by about $40(002)_{\mathrm{Au}}$ planes have been studied. For this purpose, cross sections along [010] and [110] were characterized [1,4] by HREM using a Jeol 4000EX $\left(C_{\mathrm{s}}=1.05 \mathrm{~mm}\right)$ running at $400 \mathrm{kV}$.

\subsection{Distortion Measurement}

HREM cross-section images of multilayer are recorded and then digitized with a CCD camera. The basic method of the image analysis is to localize very accurately the bright dots (atomic column) from the HREM micrographs. The lattice distortion in the growth direction $z$ is then extracted by measuring the displacement of the dots with respect to a reference lattice taken in the undistorted Au layer. The method described in [5] has been used mainly in semiconductor multilayers $[5,6]$. It has to be noticed that in the original method the authors used a Fourier filtering to enhance the image contrast which is generally not suitable for highly strained multilayers as in our case.

In our case, we determined the accurate positions of the intensity maxima by a recursive centre of mass extraction $[1,6]$. The displacement profile $u\left(z_{n}\right)$, with respect to the undisturbed $\mathrm{Au}$ lattice, is measured as a function of atomic plane number $n$. The difference $\delta=u\left(z_{n}\right)-u\left(z_{n}+1\right)$ between two adjacent planes gives the local distortion:

$$
\delta=d_{002}^{\mathrm{mes}}-d_{002}^{\mathrm{Au}}
$$

where the $d$ 's are the distances between the (002) planes measured in the distorted region and in the $\mathrm{Au}$ reference lattice. The displacements were measured for each dots and then averaged along the 002 planes in order to reduce noise. Strain profiles in the growth direction are thus extracted from HREM images. The experimental profiles are compared to an elastic model.

\subsection{Experimental Results}

In a simple model, one assumed i) abrupt $\mathrm{Au} / \mathrm{Ni}$ interfaces, ii) the Au layers thick enough to be considered as an undistorted substrate for each Ni layers and iii) in the Ni layers, $a$ homogeneous biaxial stress field imposed by the Au substrate: $\varepsilon_{11}=\varepsilon_{22}$ in the $(x, y)$ growth plane $\left(x, y, z\right.$ being the $\{001\}$ directions) and a free surface $\sigma_{33}=0$. Using elastic theory [8], one ends up with a tetragonal distortion of the Ni lattice:

$$
\varepsilon_{33}=-\frac{2 C_{12}}{C_{11}} \varepsilon_{11}
$$

$\varepsilon_{11}=\left(d_{200}^{\mathrm{Au}}-d_{200}^{\mathrm{Ni}}\right) / d_{200}^{\mathrm{Ni}}=15.6 \%$ with respect to bulk Ni. The values of elastic constants $C_{i j}$ are extracted from [7]. Figure 1 compares one experimental profile obtained across a $\mathrm{Au} / \mathrm{Ni}$ 


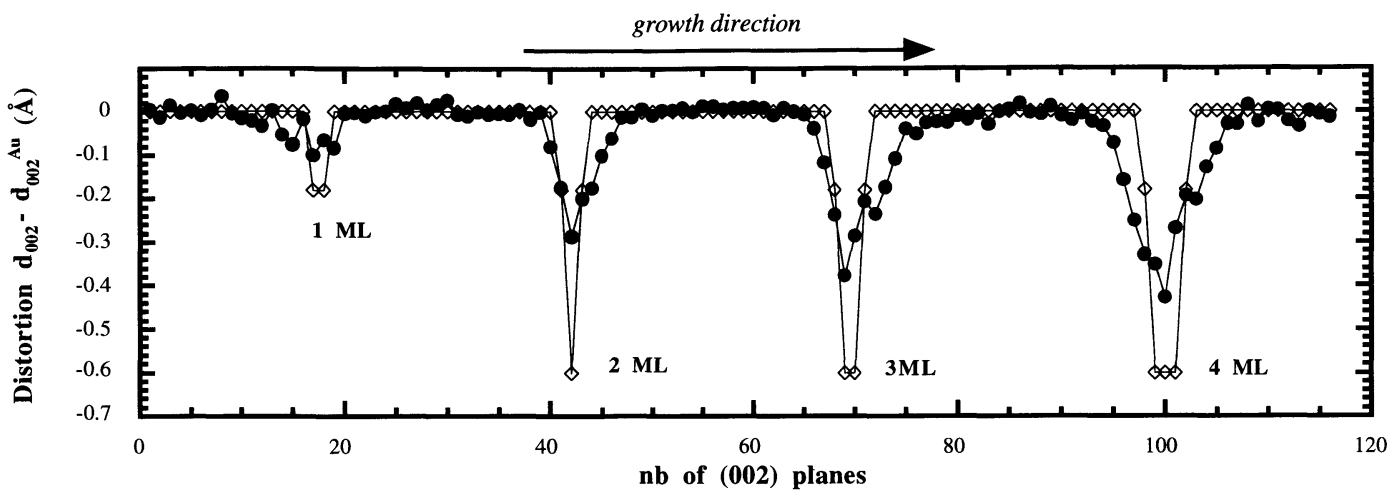

Fig. 1. - Distortion profiles in a $\mathrm{Au} / \mathrm{Ni}$ multilayer with increasing $\mathrm{Ni}$ thicknesses ranging from 1 to $4 \mathrm{ML}$. In black dots, experimental measurements and white dots the expected values extracted from a simple elastic model (see text).

multilayers and superimposed the expected values from (2). It appears that the measured distortions are far less than the expected ones deduced from elasticity theory. Moreover, these profiles are extended over a number of (002) planes larger than the nominal number of evaporated $\mathrm{Ni}$ planes. They have also an asymmetrical shape, the second interface being wider. The reliability of the shape of experimental strain profiles has been checked [1]: an abrupt and an extended profiles can be unambiguously discriminated by HREM imaging and thus experimental $\mathrm{Ni}$ profiles are definitively asymmetrical. Following Matthew's theory [8] which described the epitaxy of an layer on a substrate, the critical thickness of a Ni epilayer on [001]Au, for which the strain relaxation occurs, must be less than 1 ML. Nevertheless despite this misfit, an epitaxial growth of Ni takes place until a nominal Ni thickness of $4 \mathrm{ML}$. In fact the mixing reduces the misfit between $\mathrm{Au}$ and $\mathrm{Ni}$ and allows a stress relaxation between the layers. The solubility between $\mathrm{Au}$ and $\mathrm{Ni}$ at room temperature is less that 5 at\%. Nevertheless, segregation of $\mathrm{Au}$ atoms on the $\mathrm{Ni}$ surface during the deposit and interdiffusion induced by the stress allows to explain the spreading of the chemical profiles. This has been already mentioned in semiconductors [9]. As a consequence, epitaxy of $\mathrm{Ni}$ on $\mathrm{Au}$ can take place until a pseudo-critical thickness larger than the expected one.

In order to obtained these results (presented with more details in [1]), we had to take into account the effects of experimental parameters on the analysis. We are going now to detail this. We noticed that the location of the areas of investigation along a fixed layer with respect to the specimen edge gives rise to different shapes of the distortion profiles. This shows a strong dependence of the specimen thickness on the measurement. This is presented in Figure 2 which shows one HREM image of a $\mathrm{Au} / \mathrm{Ni}$ multilayer [011] cross section and the different areas where the distortion profiles were determined. The number of Ni layer varies from 1 to $4 \mathrm{ML}$ along the growth direction. No dislocation is present, the contrast variation comes only from the distortion introduced by $\mathrm{Ni}$ layers in $\mathrm{Au}$. Figure $2 \mathrm{~b}$ shows the corresponding measured profiles for each areas marked on the image. The edge of the sample (the thinnest part) is on the left. It can be clearly shown that the distortion values decrease as the thickness of the sample decreases: the distortion profiles have been found to be very flat for small thicknesses (left hand side) showing that the strain seems to be entirely relaxed. They remain stable up to a given specimen thickness. It is important to point out that the chemical contrast between $\mathrm{Au}$ and Ni layers is very weak and even doesn't exist for very thin areas. 

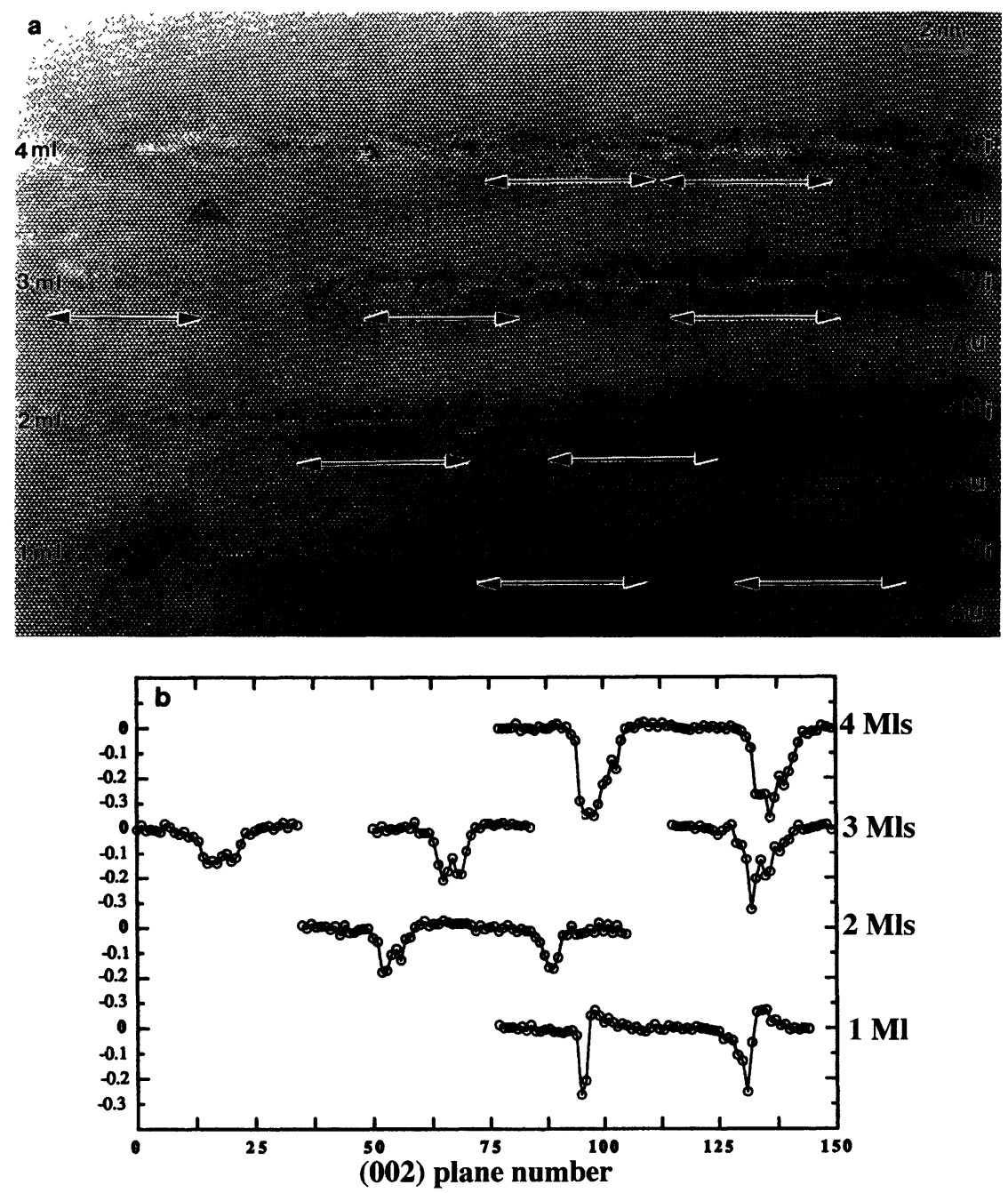

Fig. 2. - a) HREM image of a Au/Ni multilayer containing 1, 2, 3 and $4 \mathrm{Ni}(002)$ planes embedded in 40 (002)Au planes. The arrows show the areas where the distortion profiles (b) were measured. The specimen edge is on the left. Jeol 4000EX, $C_{\mathrm{s}}=1.05 \mathrm{~mm}$.

Thus in order to understand the behaviour of distortion profiles extracted from HREM, one has to explore the influence of different experimental parameters: thickness dependence (combined with defocus) of HREM images and the effect of sample preparation...

\section{Influence of Sample Thickness and Microscope Defocus}

It is well known that HREM images - i.e. position of the black and white dots on the image - vary with the specimen thickness and the defocusing distance. Thus it is of importance to firstly investigate the influence of both parameters on the distortion profiles. This will be done on image simulations [10]. For that purpose, images of 3 different asymmetrical composition gradients a, b and c (Fig. 3a) of $\mathrm{Ni}$ compound embedded between two Au layers have been 
simulated as function of specimen thickness and defocus. We chose to simulate asymmetrical profiles because of the shape of the experimental ones.

The profiles a and b correspond to a total amount of $\mathrm{Ni}$ of 4.2 and 3.6 ML respectively spread over 12 (002) planes with a maximum concentration of $70 \%$ and $60 \%$ on the 4 th plane. The profile c corresponds to $4 \mathrm{Ni} \mathrm{ML}$ with a maximum of $80 \%$ on the 8 th plane, the profile in this case is spread over 10 (002) planes. Thus the reliability of the image intensity maxima position and the profile asymmetry is checked with these profiles which correspond to a $\mathrm{Ni}$ amount around $4 \mathrm{ML}$.

Figure $3 \mathrm{~b}$ shows the distortion profiles extracted from the HREM images simulated in each cases for three different thicknesses $(7,10$ and $16 \mathrm{~nm})$ and different defocusing conditions as indicated in the frames. The imaging conditions were the following: $400 \mathrm{kV}, C_{\mathrm{s}}=1.05 \mathrm{~mm}$, defocus spread $=7 \mathrm{~nm}$, beam divergence $=0.7 \mathrm{mrad}$, objective aperture diameter $=14 \mathrm{~nm}^{-1}$. It has to be noticed that the defocusing distances were chosen around $-70 \mathrm{~nm}$ which in fact gives a maximum in intensity on the gold columns (these being imaged in white) for a wide range of thicknesses $[1,4]$ and which corresponds to the common experimental conditions.

For thicknesses lower than $16 \mathrm{~nm}$, and with a defocus around $-70 \mathrm{~nm}$ the intensity maxima are well localized on the atomic column except for sharp strain variation (i.e. Ni concentration) as illustrated by profile c. For larger thicknesses $(16 \mathrm{~nm})$, a shift between the real position of the column and the intensity maxima in the image is observed. This displacement due to Fresnel effect is a limitation for the estimation of local chemical composition and we can notice that this effect is more pronounced when the composition varies rapidly (sharp interface in $c$ profile). On the other hand, in the case of a small composition gradient, the fringe spacing is quite accurately reproduced up to a $16 \mathrm{~nm}$ thickness and is stable around the $-70 \mathrm{~nm}$ defocus.

In conclusion one can say that the shape and the expansion of the measured profile is qualitatively representative of the real structure of the interfaces (sharp or abrupt), but limited experimental conditions have to be used to be able to extract a reliable local composition with the help of linear elasticity. These conditions allowed the authors $[1,4]$ to extract reliable chemical profiles in $\mathrm{Au} / \mathrm{Ni}$ multilayers containing an increasing number of $\mathrm{Ni}$ layers and to find experimentally the total amount of $\mathrm{Ni}$ in each layer.

But these simulations do not explain why in the thinnest parts of the specimen, there is no chemical contrast and that the distortion profiles seem to be relaxed. We have now to take into account other experimental parameters to discuss this phenomenon: the electron beam interaction with the sample and specimen preparation.

\section{Influence of a Mixed Layer on Both Surfaces}

\subsection{Influence of the Incident Electron Beam}

The $400 \mathrm{keV}$ electrons might introduced irradiation damages such as atom displacements during the observations. The threshold energy are $1300 \mathrm{keV}$ for $\mathrm{Au}$ and $440 \mathrm{keV}$ for Ni [11]. The value for $\mathrm{Ni}$ is only slightly higher than the incident beam energy value. Unlike in [12] where $\mathrm{Ni} / \mathrm{C}$ multilayers were observed, no change in the specimen structure or contrast was detected during the observations. Thus we can considered that the electron beam has no effect on the $\mathrm{Au} / \mathrm{Ni}$ multilayers.

\subsection{Influence of the Ion-Milling: HREM Simulation and Profiling}

On the other hand, $\mathrm{Ar}^{+}$ions accelerated at 4 to $5 \mathrm{kV}$ were used for final thinning of the specimen (Gatan, Duo-Mill). The effect of ion-milling on the Ni/C multilayers has been clearly 

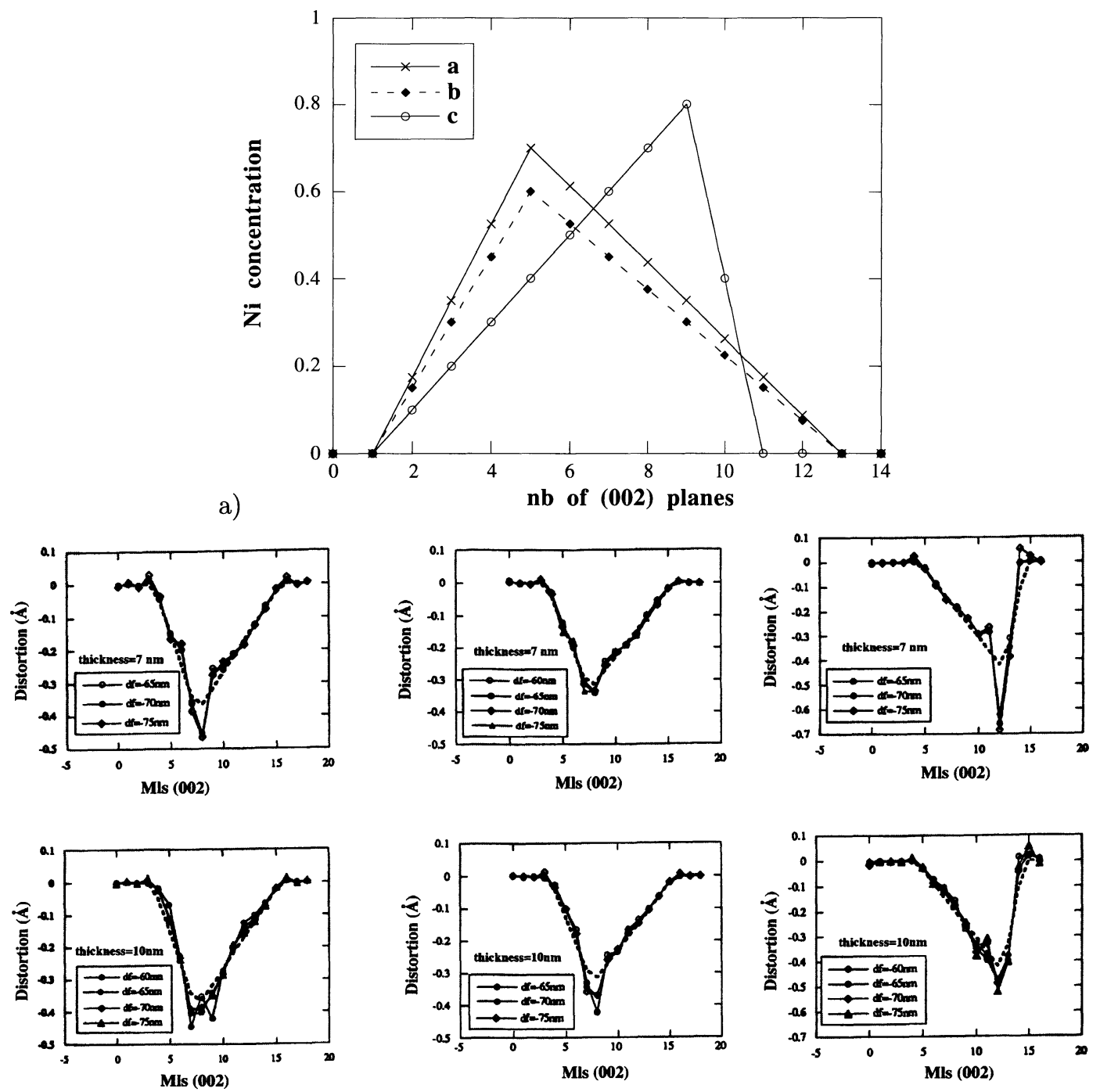

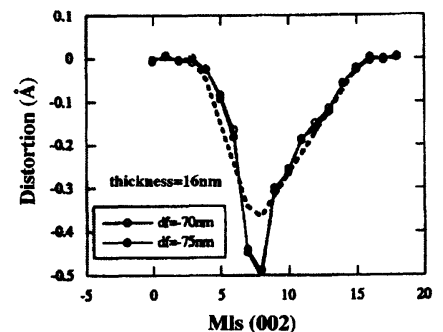

Profile a

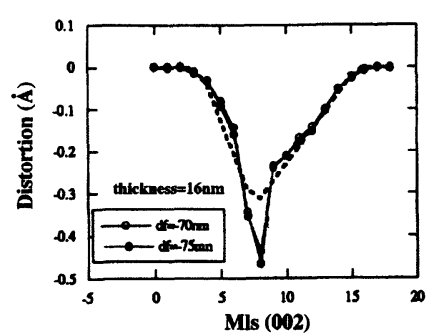

Profile b

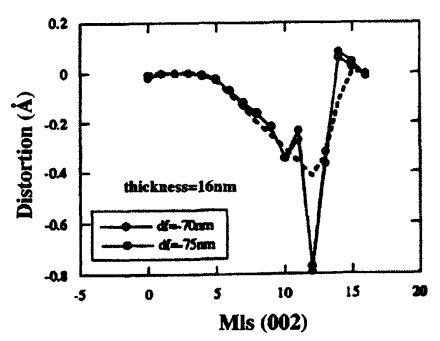

Profile c

b)

Fig. 3. - a) Three Ni theoretical concentration profiles used in image simulations. b) Distortion profiles extracted from HREM simulated images of the three $a, b$ and $c$ Ni profiles. Three different specimen thicknesses $(7 \mathrm{~nm}, 10 \mathrm{~nm}$, and $16 \mathrm{~nm})$ are checked and the defocusing distance varies around an underfocus of $-70 \mathrm{~nm}$. 
shown in [12] by comparison with cleaved specimens. The structure of the Ni layer and the periodicity of the multilayer were shown to be largely affected. In our case, the multilayer periods measured by HREM and X-rays are similar. The extension of the distortion profiles has been confirmed by X-rays diffraction and numerical calculations (Monte Carlo) [13].

Thus, it seems that the major effect is due to atom displacement giving rise to a mixed surface layer which might be amorphous or crystalline. We rejected the amorphous layer hypothesis because no amorphous layer was detected on the specimen edge. HREM image simulations of $\mathrm{Au} / \mathrm{Ni}$ interfaces for thin specimen thicknesses showed that a chemical contrast should be present for thin areas. According to these results, we concluded that the lost of chemical contrast between $\mathrm{Au}$ and $\mathrm{Ni}$ for thin areas is due to a mixture occurring during the ion-milling.

The thickness of this disturbed layer was determined by the following procedure. First, the thickness of the specimen as a function of position with respect to the specimen edge was determined: the sample was tilted around the [01-1] direction (perpendicular to the growth direction and contained in the growth plane) and the enlargement of the projection of the $\mathrm{Ni}$ layer was measured for different tilting angles. Then, the limit thickness for which the distortion profile is stable has been chosen as the thickness for which a complete mixture between the two components occurs. This thickness was determined to be around $2 \mathrm{~nm}$ which was assumed to correspond to two equal mixed $1 \mathrm{~nm}$ thick layers on each side of the sample for thicker areas.

We then simulated the influence of two such layers assuming that they are made of a homogeneous solid solution of $\mathrm{Au}$ and $\mathrm{Ni}$ atoms. The mixing is assumed to take place only on the profile extension p. Figure 4a shows the two conditions used for the simulations: a non perturbed sample with the $\mathrm{Ni}$ profile presented in Figure $2 \mathrm{a}(\mathrm{c})$ and a perturbed one with on both side a $1 \mathrm{~nm}$ thick perturbed layer with a mean concentration. Figure $4 \mathrm{~b}$ shows the HREM image simulations for one defocusing distance $(-70 \mathrm{~nm})$ and for different thicknesses $(4.5 \mathrm{~nm}$, $6 \mathrm{~nm}, 9.0 \mathrm{~nm}, 13.3 \mathrm{~nm})$. The left images show an undisturbed gradient and the right images the effect of a $0.9 \mathrm{~nm}$ thick mixed layer on each side of the sample.

Contrast variation can be observed for small thicknesses but the effect is better shown on the corresponding distortion profiles (Fig. 5). It is clear that the mixed layer has the greatest influence in the thinnest part of the specimen $(t=4.5 \mathrm{~nm})$. Up to a total thickness of about $10 \mathrm{~nm}$, the measured profile no longer corresponds to the real profile. For a larger thickness the influence of the mixed layers becomes negligible.

The model we used to simulate the mixed layer is very crude: we assume that there is full incoherence between the three different layers, mixed, unmixed, mixed. This might enhance the difference between the disturbed and undisturbed profiles. Some more precise details might be introduced in the model. Nevertheless the experimental results are qualitatively correctly reproduced.

\section{Influence of the Two Free Surfaces of the TEM Sample}

Due to the thinning, the TEM specimen has two free surfaces which might modify the elastic strain distribution with respect to a bulk sample: bent of the planes close to the free surfaces and variation of the interplanar spacing in the growth direction. The strain distribution in the whole specimen could be calculated using the formulae given in [2]. However, a first approximation using limit conditions can be used to estimate this influence.

Considering a pure $\mathrm{Ni}$ specimen with two free surfaces, we assume an homogeneous uniaxial tension $\left(\varepsilon_{11}=m \neq 0\right)$ along the axis in the growth plane which does not correspond to the free surfaces of the sample, and free surface conditions for the other in-plane direction and the perpendicular growth direction $\left(\sigma_{22}=\sigma_{33}=0\right)$. Depending on the type of the cross section 

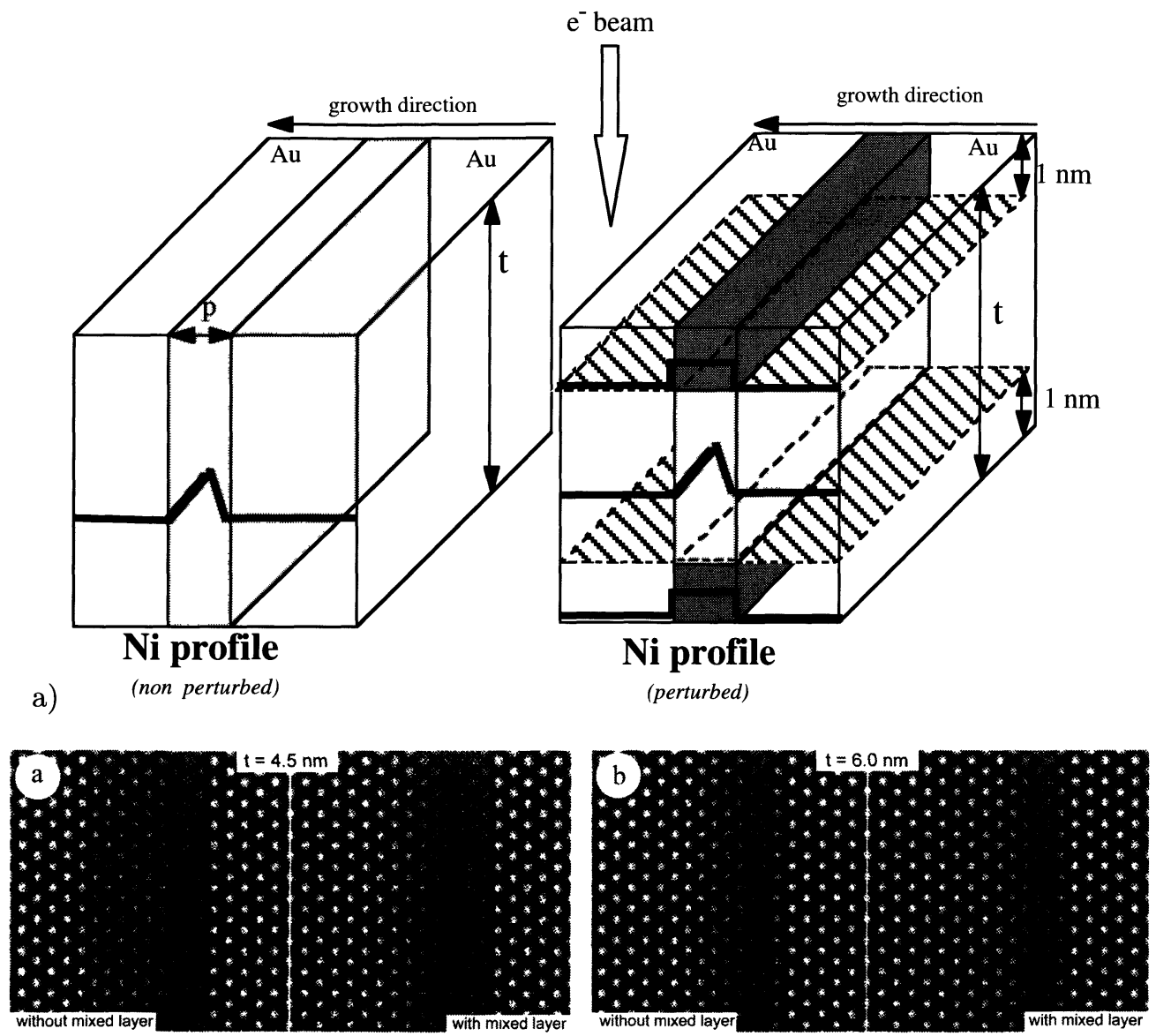

b)
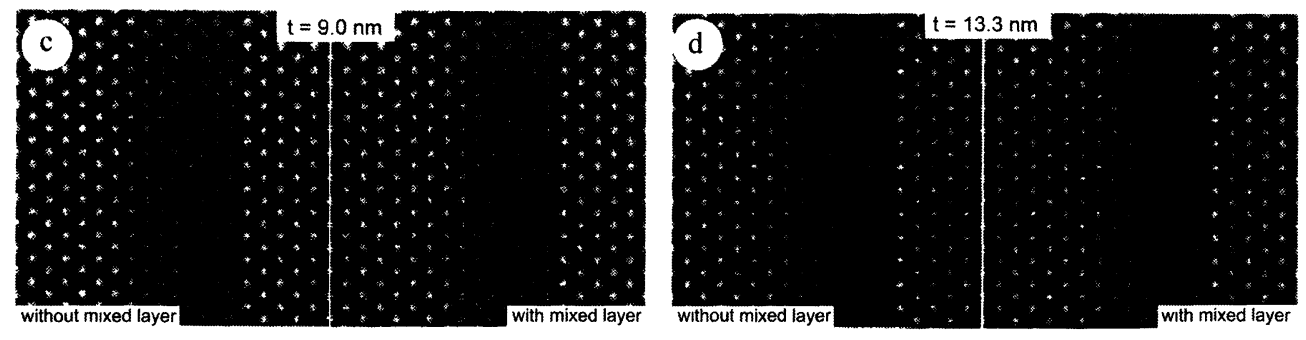

Fig. 4. - a) Drawing of the 2 conditions used for HREM simulations: non perturbed and perturbed samples i.e. covered on both sides with a $1 \mathrm{~nm}$ thick uniform mixed $\mathrm{Au} / \mathrm{Ni}$ layer. The Ni profile across the layers is shown: asymmetrical profile c (Fig. 2a) in the undisturbed layer and constant composition in the disturbed layers. b) HREM image: the total thickness $t$ varies as indicated a) $4.5 \mathrm{~nm}$, b) $6.0 \mathrm{~nm}$, c) $9.0 \mathrm{~nm}$, d) $13.3 \mathrm{~nm}$. The defocusing distance is $-70 \mathrm{~nm}$.

specimen the in-plane directions are $\{010\}$ or $\{110\}$. The transverse strain along the $z$ growth direction will be respectively

$$
\varepsilon_{33}^{010}=-\frac{C_{12}}{C_{11}+C_{12}} \varepsilon_{11}
$$



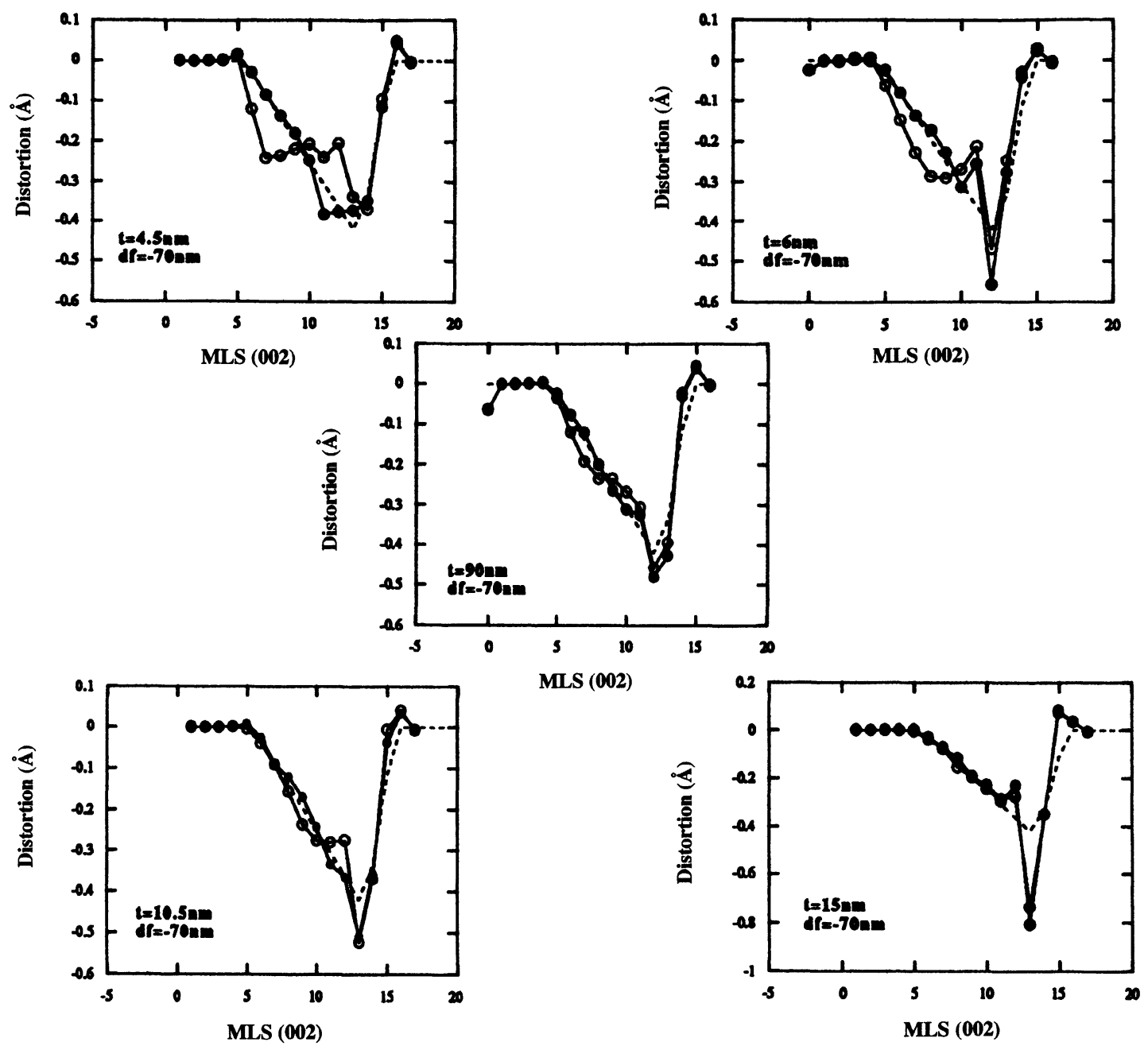

Fig. 5. - Distortion profiles extracted from HREM simulated images taking the conditions described in Figure 4, as a function of the total sample thickness $t$. The black dots correspond to the undisturbed case and white dots show the effects of a $1 \mathrm{~nm}$ thick perturbed layer on each side.

or

$$
\varepsilon_{33}^{110}=-\frac{4 C_{12} C_{44}}{2 C_{12}^{2}-C_{11}^{2}-2 C_{44} C_{11}-C_{12} C_{11}} \varepsilon_{11}
$$

with bulk Ni elastic constants.

In the isotropic case, $2 C_{44}=C_{11}-C_{12}$, the two formulas would have led to the same relationship for the two different crystallographic directions, the ratio $\varepsilon_{33} / \varepsilon_{11}$ being given by the Poisson's ratio. But, the isotropic conditions cannot be applied in our case.

Table I reports the distances between the (002) planes given by the three formulas (2-4) and for comparison, the bulk $\mathrm{Ni}$ (002) distance and the mean (002) parameter of the multilayer [2] in the case of $4 \mathrm{Ni} \mathrm{ML}$ embedded in $40 \mathrm{Au} \mathrm{ML} d_{0}=\left(m a_{1}+n a_{2}\right) /(m+n)$ where $m$ and $n$ are the numbers of monolayers for the materials of parameter $a_{1}(\mathrm{Au})$ and $a_{2}(\mathrm{Ni})$ respectively. 
Table I. - (002) interplanar distances for different structural conditions.

\begin{tabular}{|c|ccccc|}
\hline & $\begin{array}{c}40 \mathrm{Au} / 4 \mathrm{Ni} \\
\text { mean } \\
\text { parameter } d_{0}\end{array}$ & $\begin{array}{c}\text { bulk } \\
\text { fcc Ni }\end{array}$ & $\begin{array}{c}\text { Tetragonal } \\
\text { distortion (2) }\end{array}$ & $\begin{array}{c}\text { thin film } \\
{[001] \text { free }} \\
\text { surface (3) }\end{array}$ & $\begin{array}{c}\text { thin film } \\
{[110] \text { free }} \\
\text { surface (4) }\end{array}$ \\
\hline$d_{002}^{\mathrm{Ni}}(\mathrm{nm})$ & 0.202 & 0.176 & 0.144 & 0.166 & 0.158 \\
\hline
\end{tabular}

One has to notice that the relaxation (with respect to the fully tetragonally deformed $\mathrm{Ni}$ ) introduced by a (001) free surface is larger than by a (011) free surface. As our measurements are made on [011] cross section images, surface relaxation effect, and as a consequence its influence on the strain measurements, is in any case lower than for [001] observations.

Gibson and Treacy [2] calculated the strain in superlattices with periodic composition variation in the growth direction. Following Cahn's approach [14] they investigated the influence of thin film condition on the Fourier components of the distribution. This was done for isotropic conditions and assuming similar elastic properties for the two materials. Their main result is that the stress is mainly localized at the interfaces and consequently thin film relaxation occurs only far from the interface if the layers are thick enough. Thus, the relaxation is negligible at the interfaces if the specimen thickness is much larger than the width of the layer in the growth direction.

Thus, the thin film relaxation is negligible for a very thin layer embedded and stressed between two thick layers of an other material. The stress can be considered as biaxial and leads to the expected tetragonal strain calculated in bulk conditions. In our case, the mean parameter $d_{0}$ of the $(40 \mathrm{ML}) \mathrm{Au} /(1$ to $4 \mathrm{ML}) \mathrm{Ni}$ multilayer is similar to the gold parameter (misfit 1\%). Thus we deal only with strain in Ni layers which are far thinner than the Au layer. In these conditions, it might be assumed that the relaxation in Ni layers due to thin film effect is negligible. This conclusion was a posteriori verified by atomistic simulations of our $\mathrm{Au} / \mathrm{Ni}$ multilayers with free surfaces ( $\mathrm{T}$. Deutsch, private communication). No relaxation introduced by the thin film condition was detectable numerically.

\section{Conclusion}

Quantitative analysis done on HREM micrographs and simulated images have shown the effect of the experimental parameters (sample thickness and microscope defocus) on the distortion profile. It has been shown that the shape of the profile is preserved but limited conditions have to be used for a chemical interpretation. The most limiting factor is the introduction of a mixed layer by ion-milling: quantitative analysis cannot be done on too thin areas. It has also been shown that the effects of thin film relaxation are negligible for these multilayers.

The authors would like to emphasize that the assertions of the present paper are only valid for the present observations and that the response of other materials might be quite different. Thus the experimental conditions of preparation and their influences on the HREM images interpretation have to be checked carefully for each particular case if the goal is to extract quantitative results from HREM pictures. 


\section{Acknowledgments}

Special thanks are due to B. Gilles and A. Marty who prepared the MBE multilayers, to T. Deutsch and F. Lançon who performed numerical relaxations and to J.L. Rouvière for his help in image processing. This work was partially supported by EEC contract $\mathrm{n}^{\circ}$ : SC1*-CT910703(TSTS).

\section{References}

[1] Bayle P., Deutsch T., Gilles B., Lançon F., Marty A. and Thibault J., Ultramicroscopy 56 (1994) 94.

[2] Gibson M. and Treacy M., Ultramicroscopy 14 (1984) 354; Treacy M. and Gibson J., J. Vac. Sci. Technol. B4 (1986) 1458.

[3] Gilles B., Marty A., Patrat G., Vassent J.L., Joud J.C. and Chamberod A., MRS, Mechanisms of Thin Film Evolution (Boston-1993).

[4] Bayle P., PhD Thesis, Université Joseph Fourier, Grenoble (1994).

[5] Bierwolf R., Hohenstein M., Phillipp F., Brandt O. and Crook G.E. and Ploog K., Ultramicroscopy 49 (1993) 273.

[6] Jouneau Ph., Tardot A., Feuillet G., Mariette H. and Cibert J., Inst. Phys. Conf. 134 (1993) 329.

[7] Hirth J.P. and Lothe J., Theory of Dislocations (Mac Graw Hill, New-York, 1968) p. 25.

[8] Matthews J.W. and Crawford J.L., Thin Solid Films 5 (1970) 187.

[9] Dandrea R. and Duke C., Phys. Rev. 45 (1992) 14065.

[10] Stadelmann P.A., Ultramicroscopy 21 (1987) 131.

[11] Urban K., Phys. Stat. Sol. A56 (1979) 157.

[12] Scholz R., Electron Microscopies of Boundaries and Interfaces in Materials Science, J. Heydenreich, W. Neumann, Eds. (Max-Planck Inst., Halle, 1994) p. 264.

[13] Deutsch T., Bayle P., Lançon F. and Thibault J., J. Phys.: Condens. Matt. 7 (1995) 6407.

[14] Cahn J., Acta Met. 9 (1961) 795; 10 (1962) 17925 (1977) 1021. 